\title{
MedienPädagogik
}

www. medienpaed.com

Zeitschrift für

Theorie und Praxis

der Medienbildung

ISSN 1424-3636

Themenheft Nr. 24: Educational Media Ecologies

\section{Visual Storytelling in Career Counselling - An Ecological Approach}

Heinz Moser and Thomas Hermann

\begin{abstract}
In this paper, we present the concept of the project «Visualized vocational aspirations: Potentials of photography for career counselling and vocational preparation», which is funded by the Swiss National Science Foundation. The research project is a cooperation between the "Zurich University of Teacher Education» («Pädagogische Hochschule Zürich») and the "Laufbahnzentrum» (Centre of Vocational Counselling) Zürich. Vocational education often is largely language-based, which could be a problem for the lower achieving students. Visual stimulation could be a means to support the learning process of this group of at risk learners.
\end{abstract}

\section{An ecological approach of narrative career education}

First of all, the idea for this project came from media educators at the Zürich University of Teacher Education. We felt that for young people of the $21^{\text {st }}$ century the field of vocational or career counselling is embedded in an environment where media play an increasingly dominant role in the development of lifestyles and the behavioural patterns of individuals. The planning of their lives and their vocational aspirations are not independent from the emergence of a society in which computers and audio-visual media define new cultural lifestyles. In our project we want to encourage activities in which media play a more important role in the process of career education (taking photographs, illustrating career aspirations, digital storytelling etc.).

In the past the paradigm of counselling usually was based on vocational tests as the main instrument of an analytical assessment of the client. In recent decades Mark L. Savickas has advocated a change from the «scores» to the «stories»: «From this perspective, a subjective career is a reflexive project that transforms individuals from actors of their career to subjects in their own career story» (Savickas 2002 , 152). As a consequence postmodern approaches are including step-by-step communicative activities and methods such as storytelling in the process of counselling. Mary McMahon explains: "Thus counsellors engage as co-authors with the storytellers in snegotiating stories that must take into account both the individual's life and the ecological context»» (McMahon 206, 17). In this constructivist approach counselling processes are interpreted in a holistic view of individuals as being contextually located. 
In light of these developments in career counselling we found it useful to reflect about connections to the concept of media ecology as it is expressed by a definition of Lance Strate: «lt is the study of media environments, the idea that technology and techniques, modes of information and codes of communication play a leading role in human affairs» (Strate 1999). This definition shows that in this approach, the technological aspects of media are embedded in a communicative or an environmental setting. As Larry Sullivan writes in the SAGE Glossary of Social and Behavioral Science, «Media Ecology is best understood as an approach, rather than a strict ideology, to understanding the media as environments. Central to media ecology is the idea that media environments, such as books, radio, film, television, and the like, involve subtle, implicit, and informal dynamics and complex message systems» (Sullivan 2009, 315). In our project, the connection between image and narration should be expressed by taking photographs that capture the wishes and aspirations in the vocational field.

\section{Overcoming the conservative bias of media ecology}

The point made in the SAGE Glossary that media ecology should be more than an ideological approach is very important, because one of the founders of the concept of media ecology is Neil Postman: For Postman media ecology is a means to look at how media of communication affect human perception, understanding, feeling and value. And he is interested in analysing how our interactions with media facilitates or impedes our changes of survival (Postman 1970). In his keynote address at the inaugural Media Ecology Association Convention from 2000 Postman comments frankly: «From the beginning, we were a group of moralists. It was our idea to have an academic department that would focus its attention on the media environment, with a particular interest in understanding how and if our media ecology was making us better or worse» (Postman 2000, 11).

As we know, Postman is not only a moralist but also takes a conservative position on media education. He argues against television as a medium, warning that we are "amusing us to death» (as one of his bestselling books is titled). He tries to demonstrate how the content of the printing press in America was once, «coherent serious and rationals and how, under the governance of television, it has become «shrivelled and absurd - equally absurd as the TV content that have destroyed the traditional childhood.

If we refer in our project to the concept of media ecology, we are not following this conservative path. Reflecting the influences of the media does not mean that we are criticising their influence on vocational choices - for example the influence of casting shows or role models and idols. And we are not discussing the fact that lifelong vocational activities today have often been replaced by short-time jobs.

For us, it is more important that media will be seen in media ecology as a complex message system, linking human beings with media, social relationships and per- 
sonal feelings and emotions. The media system is a very important factor in the interplay of these elements and their linkages; it structures - as Postman noticed - what we can see and say, and therefore, do. In a similar way McLuhan speaks of the media ecology as the way in which media change our perceptions of the world. Media ecology has taken up the task of detailing some of the many ways this has happened and will continue to happen as new media like computers, internet and Facebook are influencing our lives (Levinson 2000, 17). It is «synaesthesia», the balanced interplay among all of the senses, which is the effect of hypermedia. "Hypermedia» in this sense are part of the contemporary digital culture. We agree with James C. Morrison's statement: «Here, I am referring not to a collection of media that might be invoked with hyperlinks. Rather, I am referring to the system of interlinked communications itself, to hypermedia as a means of linking both textual and audio-visual files» (Morrison 2000, 38).

\section{Career counselling - a postmodern approach}

Looking back to the field of career counselling as an approach, based on central concepts of media ecology and "synaesthesia», we developed a framework, involving the following key-points, to be adopted in our project-strategy:

1. Career Counselling is not a technical process where the counsellor searches for information by testing and interviewing in order to develop a rational decision for his clients. In contrast to such approaches counselling has to be described as a communicative process. Vance Peavy $(1997,84)$ assumes that dialogical conversation is a form of communication, which seeks common ground and does not foster the superiority of the counsellor.

Both are a part of the same common life-space. For Peavy this is the ground where the psychological and the sociological spheres meet. In these spheres the individual actions, values and knowledge are inextricably linked together (Peavy 1997, 78). Counselling should become a collaborative activity, in which the client participates actively in the search for his vocational future.

2. The coherence of the identity in social life is kept alive by stories, which give us a sense of ourself, of personal comfort and stability. As Peavy notices: «A person needs to have a coherent life story, which is constantly being revised as she moves through live» (Peavy 1997, 97). All the choices a person has to make in vocational counselling, thus, are embedded in activities that are linked to her or his own life stories. As a consequence storytelling as a method of counselling is a key-concept of our approach. Working with stories in school should help young people to clarify their vocational aspirations.

3. If we give practical expression to these essential characteristics of career counselling in the digital age, it is necessary to have in mind the situation of the youth. The minds of young people - our clients in career education - are deeply shaped by 
the influences of media. As digital natives they are born into an environment where visual stimuli are much more present than in the age of the traditional literacy. In this visual culture, understanding can be better reached if it is embedded in a learning environment which does not exclude visuality and synaesthesia as the interplay between the textual and the audio-visual. For this generation, stories are not only linguistic experiences but also imaginational expressions in the context of a visual culture - for example in cartoons, television series, YouTube videos etc. This description of the theoretical concepts of our project shows how the concept of media ecology is guiding the development and elaboration of the main ideas of our project. In the next section of this paper we describe more concretely the concept of storytelling that we realize in schools.

\section{Our concept of storytelling}

The basic definition of a story as outlined by Hoffmann (2010, $2 \mathrm{ff}$.) describes a narrative as a representation of two or more events. These are linked to one another following the properties of «narrative sequence (chronology and causality) and evaluation» (ibid. 4). Hoffmann notes that this definition is «not constrained by the use of any particular semiotic mode» (ibid. 2). Thus a series of photographs can form a narrative as well as events that are conveyed through spoken or written language.

A more sophisticated narrative structure includes criteria such as complications, solutions and transformations. While it takes some time to develop such a narrative, it certainly works well in an open setting of biographical - maybe even therapeutic - self-reflection. However, for our purpose we felt that we had to keep the story-telling task as simple and straightforward as possible, considering the relatively small amount of time teachers allotted to career counselling activities in the classroom. Furthermore, one of the major aims of the project was to give lower achieving students an equal voice and we did not want to discourage them with a complex assignment. Also, students do not need to be familiarized with elements of a story, such as characters, setting and time, since they themselves are the protagonists, acting in their everyday environment or - if they so wish-in imagined professional environments, using props etc. associated with particular professional activities.

Thus, following Hoffmann's basic definition, we asked the students to produce three or more photographs of past and present career wishes (however vague they were) which they would put together in a PowerPoint presentation, usually in a chronological order, ending on an outlook into the future, i.e. showing what they were planning to do next. Furthermore, students were asked to comment on the slides for the class, explaining what jobs they fancied at a certain time of their lives and what they found fascinating about them. Thus, the students produced 
two distinct narratives that are closely interwoven: (a) a visual narrative consisting of at least three photos (some students would produce up to 16 PowerPoint slides containing more than 20 photos); (b) a spoken narrative explaining their slides and photos. Standing in front of the class and talking about ones vocational aspirations also required some presentation skills.

\section{Photographing vocational aspirations}

The project focuses on the production of photos in vocational preparation setting at school. Students actively produce photos of their vocational aspirations to trigger off the reflection process. During one week, they take pictures of their own vocational aspirations. They document the fascinating aspects associated with their target jobs. Some pupils consulted their private photo albums for pictures representing their former vocational aspirations. Other students demonstrated their career-knowledge with pictures documenting typical activities of a certain job.

All these pictures were uploaded in digital form on a computer. During the next phase the students worked on their own biographical story about their vocational aspirations. To do this, they created their own PowerPoint presentations, which integrated textual elements with their pictures. The production of a kind of "vocational biography» is not only a technical challenge. It allowed the students to reflect their childhood aspirations and motivations. They construct their own biographies - a process which can help them to become more conscious of their own preferences and criteria for a career decision.

This describes the central idea of our project. For the realization of this kind of teaching and learning we prepared teaching materials. We plann to later integrate these materials in the general process of counselling in Swiss schools if this kind of visual storytelling will be successful. Therefore, an evaluation will compare this new visual approach with conventional methods of teaching.

\section{The methodological concept}

From a methodological viewpoint, this evaluation process is based on the concept of design-based research. This approach is appropriate for combining practical work with theoretical considerations. It is a kind of participatory action research where researchers cooperate closely with teachers. But unlike in action research, the theoretical foundation of the research process remains an indispensable element of the research process. As Wang and Hanafin write, design-based research can be characterized by five basic elements: It is (a) pragmatic; (b) grounded; (c) interactive, iterative, and flexible; (d) integrative and (e) contextual (Wang, Hanafin 2005, 7).

On the other hand this kind of research is orientated on concrete steps of social action. It is "grounded in real-world contexts where participants interact socially 
with one another, and within design settings rather than in laboratory settings isolated from everyday practice (Wang, Hannafin 2005, 9). This strong emphasis on practical change is related to the German concept of «Praxisforschung» (Moser 2012).

As in action research (see Moser 1974) design-based research is also characterized by an iterative cycle of five tasks: design, enactment or implementation, analysis, and redesign. We began our project (I) with some theoretical considerations about the visual society and function of storytelling. At the same time (II), we discussed the actual situation of career counselling in schools with our project partners from the Centre of Vocational Counselling and the colleagues who are teaching career counselling at our university. Then (III), we developed an educational concept grounded on taking photos and producing PowerPoint presentations for the teachers who are willing to cooperate, which is. These were presented and discussed in class. After (IV) conducting the lessons of visual storytelling in the classroom we evaluated the results and drew conclusions $(V)$ for the redesign of the teaching materials. The entire design with its five steps was piloted with three teachers and their classes in order to optimize the procedure for the larger study. The main study group included eleven teachers with their classes in the Germanspeaking part of Switzerland who were interested in the concept of visual storytelling. We will compare this group - a kind of «experimental» group - with ten other teachers who taught this subject in a more conventional way. The latter function as a control group. In a cyclical model of research this part of the evaluation is crucial. The results will be integrated into a textbook for career counselling at school.

A mixed-methods approach was selected for the main study. First, we conducted online interviews with the students of the 21 classes (experimental and control). The interviews were done with the online-tool "Surveymonkey» - with particular focus on general questions concerning the students' career plans. Some specific questions on the visual storytelling experience were posed only to the experimental group in order to evaluate the visual project as such.

Second, we observed and filmed the presentations of the students in their classrooms in order analyse the narrative structures of their productions. As a further focus we will analyse the visuals produced by the students.

Third, group interviews with the participating teachers were carried out after the project in order to gain further information on its success or failure.

\section{First results}

The results from the trial phase were encouraging. Students reported that they enjoyed producing the visual story. The actual presentation in front of the class was considered as more challenging, yet rewarding. The three piloting teachers 
reported that the students were highly motivated to produce stories of their past and present vocational aspirations for the future. One teacher was surprised that the project shed a new light on some of his students. "I was able to see a few students from a new point of view». Another teacher stressed the fact that the project involved parents, relatives and peers in a cooperative way. With the positive feedback from the trial phase, only minor changes were necessary for the main study.

To this date, the online questionnaires of the 21 classes have been partially analysed (11 experimental classes, $N=195 ; 10$ control classes, $N=189$ ). The sample included 384 students (212 males; 172 females). Their ages ranged between younger than twelve and older than fifteen years, with a majority of 12-year-olds $(N=149)$ and 13-year-olds ( $N=113)$. With 191 students who reported that the language spoken at home was German and 193 students with other linguistic backgrounds, the groups of students with a Swiss or migrant background were equally large.

Figure 1 shows the acceptance of the digital storytelling project by the experimental group. A majority of the students reported that the project was fun. To our surprise taking or collecting pictures was a little less popular, while working with PowerPoint was considered more positively. Not surprising, self-exposure during the presentation was not enjoyed by all students equally. However, more than half of the students reported that they thought they had learned a lot while working on the biographical portraits and a vast majority would recommend the project.

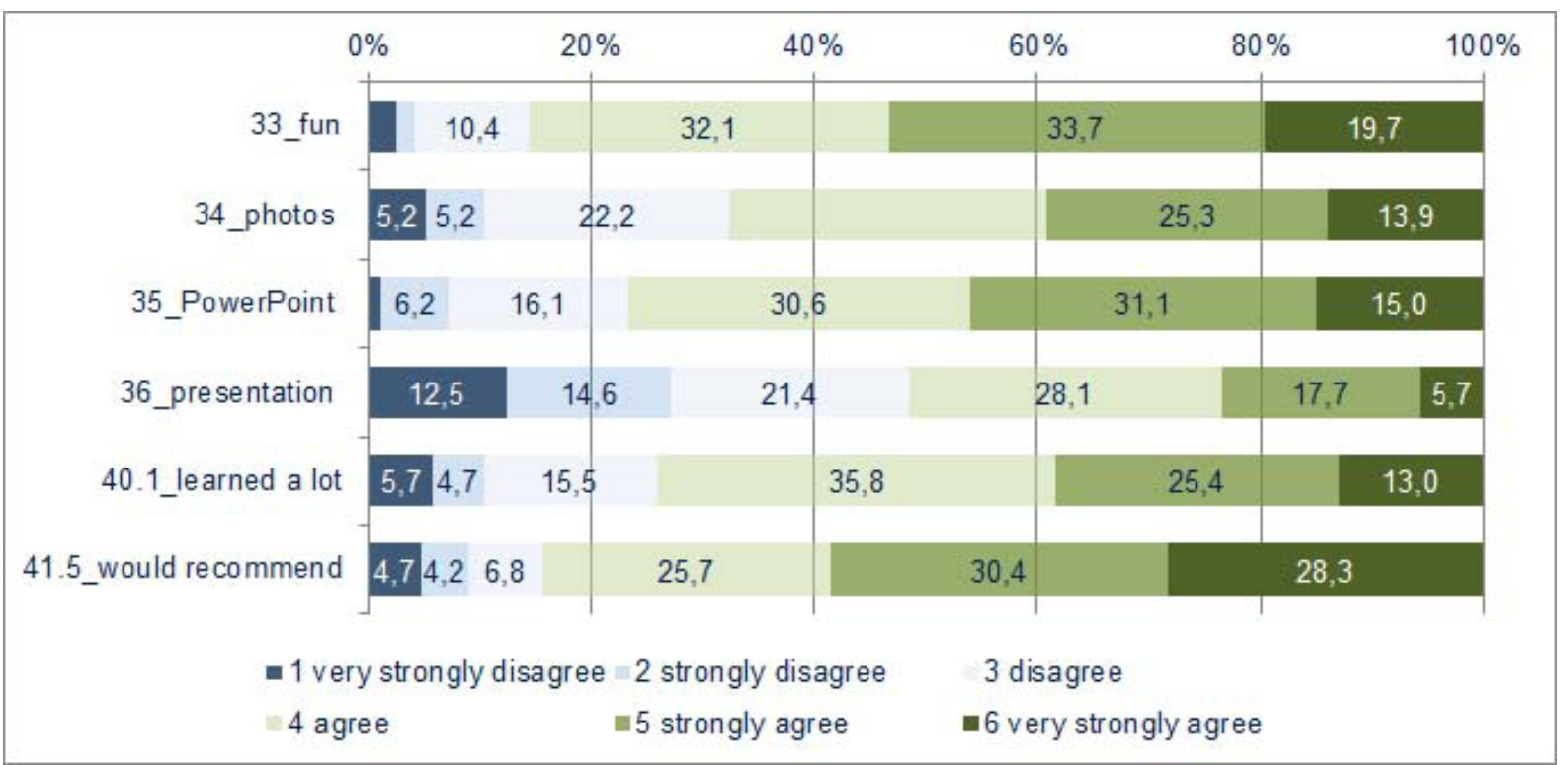

Figure 1: Evaluation of the digital storytelling project by the experimental classes $(N=195)$. 
Differences between experimental and control group need to be further analysed. The figures seem to suggest that students from the experimental group are more open to consider new job opportunities after they completed their task, whereas their peers from the control group seem to be less flexible concerning alternative aspirations.

In terms of the concept of media ecologies, one positive result reported by several teachers is that by repeating the presentations on parent-teacher-conferences, parents were (a) extremely positive about the project and (b) began to cooperate with their children and the teachers on the issue of career planning and counselling. Also, the presentations stimulated conversations among peers. Thus, the digital stories produced by the students provided a media environment with a potential to reach and involve several stakeholders in the process of career education and decision-making.

The visual and verbal data collected during the main project are currently being thoroughly analysed. Results are expected towards the end of this year (2014).

\section{Literature}

Hoffmann, Christian R. 2010. «Introduction.»In Narrative Revisited: Telling a story in the age of new media, ed. Hoffmann, Christian R. Amsterdam: John Benjamins.

Levinson, Paul. 2000. "McLuhan and Media Ecology.» Proceedings of the Media Ecology Association 1: 17-22.

McMahon, Mary. 2006. "Working with Storytellers: A Metaphor for Career Counselling.» In Career Counselling. Constructivist Approaches, ed. Mary McMahon and Wendy Patton, 16-29. New York: Routledge.

Morrison, James C. 2000. "Hypermedia and Synesthesia.» Proceedings of the Media Ecology Association 1: 37-52.

Moser, Heinz. 1975. Aktionsforschung als kritische Theorie der Sozialwissenschaften. München: Kösel.

Moser, Heinz. 2012. Instrumentenkoffer für die Praxisforschung. Freiburg: Lambertus.

Peavy, Vance R. 1997. Socodynamic Counselling: A Constructivist Perspective. Victoria: Trafford Publishing.

Postman, Neil. 1970. "The Reformed English Curriculum.»In The Shape of the Future in American Secondary Education, ed. E. A. Eurich, 160-168 New York: Pitman.

Postman, Neil. 2000. "The Humanism of Media Ecology.» Keynote Address Delivered at the Inaugural Media Ecology Association Convention Fordham University, New York, New York June 16-17, 2000. Proceedings of the Media Ecology Association 1: 10-16. 
Savickas Mark L. 2002. "Career Construction: A Developmental Theory of Vocational Behavior.» In Career Choice and Development, by Duane Brown, 149-205. San Francisco: Jossey-Bass.

Strate, Lance. 1999. "Understanding MEA.» In Medias Res 1 (1, Fall). http://www .media-ecology.org/publications/In_Medias_Res/imrv1n1.html.

Sullivan, Larry, ed. 2009. The SAGE Glossary of Social and Behavioral Science. Thousand Oaks: Sage.

Wang, Feng and Michael J. Hannafin. 2005. «Design-Based Research and Technology-Enhanced Learning Environments.» Educational Technology, Research and Development 53 (4): 5-24. 